\section{IMMUNITY}

\section{Anti-infective alarmin}

Viral and parasitic infection promotes release of the alarmin interleukin-33 (IL-33), which orchestrates protective cytotoxic $\mathrm{T}$ lymphocyte $(C T L)$ and $T$ helper type $2\left(T_{H} 2\right)$ responses.

IL-33 is expressed in the nucleus and is released after injury and necrosis, binding and signaling through the ST2 receptor to promote $\mathrm{T}_{\mathrm{H}} 2$ responses. Whether IL-33 production and release can be triggered by other mechanisms remains incompletely understood. Two recent studies provide insight into how IL-33 release is regulated and elucidate a new role for IL-33 in promoting antiviral responses.

Marsha Wills-Karp et al. (J. Exp. Med. 209, 607-622) found that infection with the parasitic helminth Nocardia brasiliensis induces lung injury and promotes the release of trefoil factor 2 (TFF2) from damaged epithelia. In turn, TFF2 induces macrophage and inflammatory dendritic cell production of IL-33, which is required to mount a $T_{H} 2$ response and control $N$. brasiliensis infection. Mice deficient in TFF2 fail to upregulate IL-33 and cannot mount an effective immune response.

In addition to promoting $\mathrm{T}_{\mathrm{H}} 2$ responses, IL-33 can also drive CTL responses and antiviral immunity. Daniel D. Pinschewer and his colleagues report that CTL expansion is impaired following viral infection in mice deficient in IL-33 (Science 335, 984-989). Productive viral replication acts as a danger signal, promoting IL-33 release from nonhematopoietic cells. IL-33 then acts directly on ST2+ CTLs, promoting their expansion and differentiation.

These studies highlight how both parasite and viral infection drive early innate immune responses and show the important part played by IL-33 in bridging innate and adaptive immune responses. $-K D S$

\section{GENETICS}

\section{Multitude effects of MCM4 mutation}

Two recent studies (J. Clin. Invest. 122, 814-820; J. Clin. Invest. 122, 821-832) discover the underlying molecular defect responsible for a variant of familial glucocorticoid deficiency, identifying it as a new DNA replication disorder and providing mechanistic insights into the disease phenotype.

In two independent studies, Claire Hughes et al. and Laure Gineau et al. identified the causative mutation in the minichromosome maintenance-deficient 4 gene (MCM4) of affected individuals. The responsible MCM4

DRUG DELIVERY

\title{
Drug delivery goes remote
}

Lack of patient compliance with treatment regimens that require daily injections or multiple medicines taken over long periods can hinder effective treatment, but help is at hand. Drug delivery from an implantable microchip wirelessly controlled by your doctor may sound like science fiction, but the first human trial of such a device is now reported by Farra et al. (Sci. Transl. Med. doi:10.1126/ scitransImed.3003276).

A microchip no bigger than a watch face was implanted subcutaneously into eight osteoporotic post-menopausal women and programmed to deliver intermittent microgram doses of the anabolic osteoporosis drug human parathyroid hormone fragment (hPTH) (1-34) once daily for 20 days. This drug is the only anabolic agent approved for osteoporosis treatment, but it requires burdensome daily injections.

The microchip is refined from an earlier prototype that was tested in dogs, allowing its use in humans. The drug is stored in individual reservoirs covered with a thin metallic membrane. When a current is applied, the membrane melts, releasing the drug. Instructions concerning dosing schedules are wirelessly transmitted to the device.

Despite the fact that a fibrous capsule had formed around the implant, the microchips produced similar pharmacokinetic profiles of hPTH (1-34) to those obtained by multiple injections. Serum concentrations of the bone formation marker type I collagen propeptide increased and were consistent with the levels reported with daily injections, serving as an indicator of increased bone formation.

The microchips were implanted in a doctor's office under local anesthetic and were well tolerated, with no toxic or adverse effects from the implant. What's next: implants delivering hundreds of drug doses per microchip and over longer periods. - DG

mutation is located in a splice site, leading to the production of a protein truncated at the $\mathrm{N}$-terminal domain. MCM4 is a component of a helicase complex required for DNA replication. Gineau et al. found no defects in the formation of this complex in fibroblasts from people with MCM4 mutations, but observed high levels of rereplication and DNA breakage in these cells, suggesting that truncation of the $\mathrm{N}$-terminal domain of MCM4 affects DNA replication and genome integrity.

Individuals with MCM4 mutation show a phenotype consistent with other DNA repair and replication disorders, with chromosomal fragility, growth retardation and a variable degree of immunodeficiency. This immunodeficiency seems to result from a natural killer (NK) cell deficiency, as Gineau et al. showed that the subjects had a selective deficiency in the CD56 dim subset of NK cells, which are the major form of mature circulating NK cells. It remains to be seen whether individuals with MCM4 mutation have an increased risk of cancer, because neither team detected any cancers in the subjects they examined. In addition, the affected individuals show adrenal insufficiency, and Hughes et al. showed that mice depleted for Mcm4 also had abnormal adrenal morphology.

The results of these two studies are particularly interesting in light of the recent identification of mutations in components of the prereplicative complex in another growth retardation disorder, Meier-Gorlin syndrome. This first identification of a human mutation in MCM4 may spur investigation into other MCM complex components as candidate disease genes. $-M S$

\section{NEURODEGENERATION}

\section{The spread of tau}

Tangles are aggregates of tau protein that propagate throughout the brain as Alzheimer's disease progresses. Two recent studies in 